\title{
Formation médicale continue: le début d'une nouvelle ère
}

Christoph Hänggeli a Werner Bauer ${ }^{b}$

a Directeur de l'Institut suisse pour la formation médicale postgraduée et continue ISFM

b Président de l'Institut suisse pour la formation médicale postgraduée et continue ISFM
Correspondance: ISFM Institut suisse pour la formation médicale postgraduée et continue Elfenstrasse 18

CH-3000 Berne 15 Tél. 0313591111 Fax 0313591112

siwf@fmh.ch
L'Institut suisse pour la formation médicale postgraduée et continue (ISFM) a créé une nouvelle plateforme de formation continue. Elle permet aux médecins de saisir en ligne leur formation continue et d'imprimer eux-mêmes le diplôme correspondant, dès qu'ils ont rempli les conditions du programme de formation continue. La procédure est également simplifiée pour les sociétés de discipline médicale: elles peuvent contrôler les données saisies par voie électronique en toute facilité, puis autoriser l'impression du diplôme. Si elles préfèrent les contrôles ponctuels, la procédure dure un peu plus longtemps. Dans tous les cas, les diplômes de formation continue sont automatiquement publiés sur www.doctorfmh.ch, ce qui rend obsolète toute attestation individuelle vis-à-vis des autorités de santé et des caisses-maladie. La charge administrative s'en trouve ainsi réduite à un minimum, tant pour les sociétés de discipline médicale que pour les médecins devant accomplir une formation continue. Dans une seconde phase, la plateforme de formation continue deviendra un registre central de toutes les offres de formation continue. Ainsi, en proposant aussi des évaluations en ligne, l'ISFM contribue fortement à la promotion de la qualité de la formation continue en Suisse.

\section{Devoir de formation continue}

Depuis l'entrée en vigueur de la Loi sur les formations médicales (LPMéd), le $1^{\text {er }}$ septembre 2007, la formation continue fait partie des devoirs professionnels réglementés par la loi. Les autorités sanitaires canto- nales chargées de la surveillance peuvent sanctionner le manquement au devoir de formation continue par un blâme ou une amende pouvant aller jusqu'à 20000 francs. La mise en œuvre du devoir de formation continue légal - notamment l'étendue et les modalités de la formation continue - incombe aux organisations professionnelles. Conjointement avec 45 sociétés de discipline médicale, l'ISFM propose un diplôme de formation continue reconnu par les autorités sanitaires et les assureurs-maladie. Il existe dans chaque domaine (uniquement titre de spécialiste) un programme de formation continue qui règle en détail les conditions d'obtention du diplôme. Le choix parmi les 45 programmes de formation continue relève de la responsabilité de chacun. Il est recommandé de choisir un programme de formation continue qui correspond le plus à l'activité professionnelle principale du moment. Le devoir de formation continue commence dans l'année suivant l'obtention du titre de spécialiste ou le début de l'activité médicale en Suisse. Les médecins qui se trouvent en formation postgraduée en vue d'un titre de spécialiste ne sont pas soumis à cette exigence.

\section{Formation continue essentielle spécifique et formation continue élargie}

Le nombre d'heures de formation continue conseillé par an (fig. 1) est de 80 heures (= crédits). Les médecins doivent attester 50 crédits par an ou 150 crédits

\section{Figure 1}

Structure des 80 heures de formation continue exigées par année.

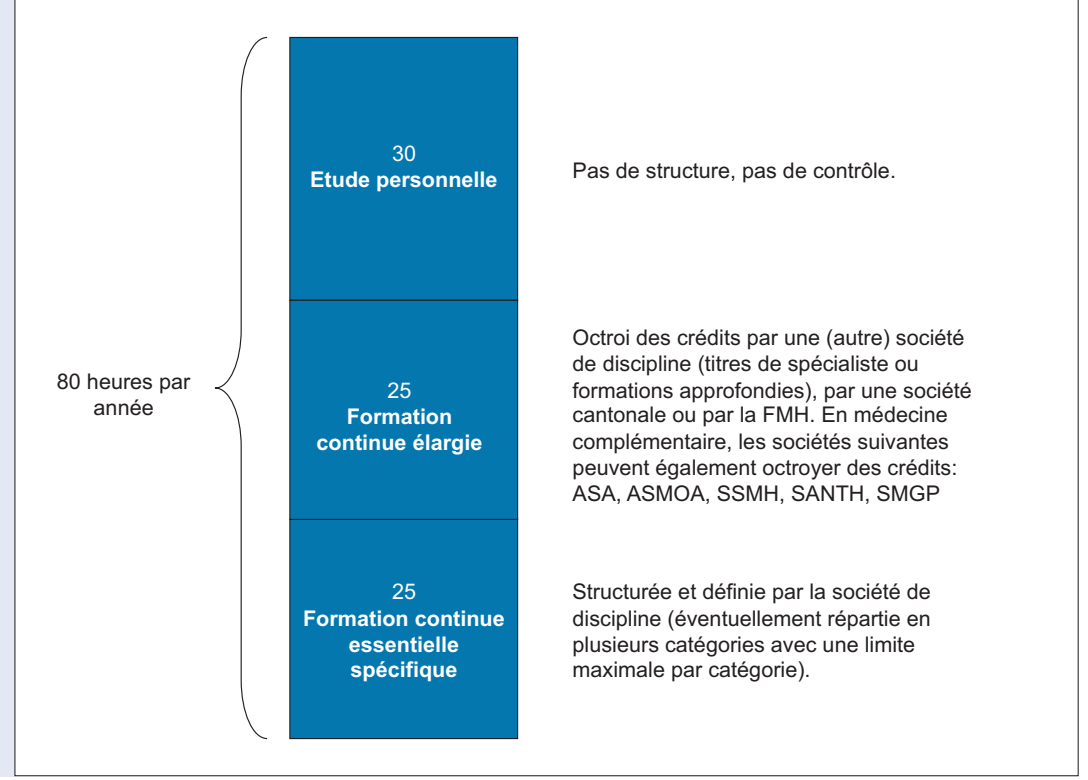


Figure 2

Page d'accès à la plate-forme de la formation continue.

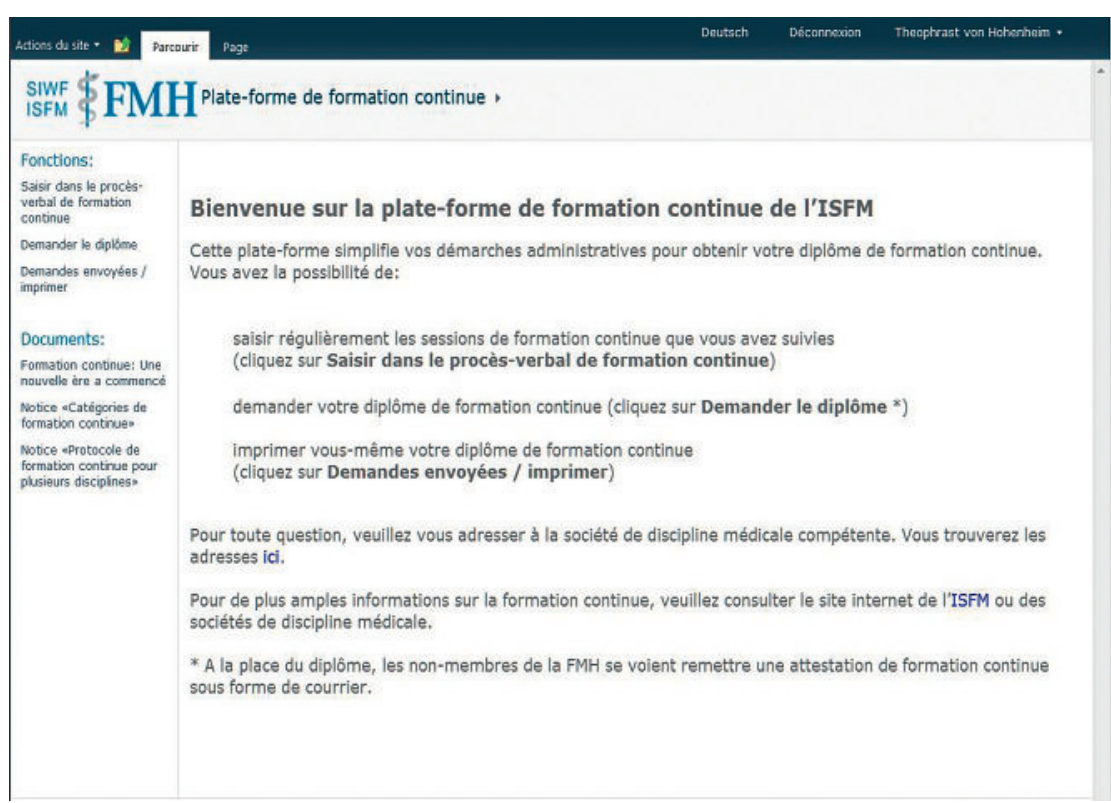

Formation continue pour les prestations de droits acquis? [1]

Six ans après l'entrée en vigueur du TARMED, 12000 médecins font encore valoir 1,3 million de positions de droits acquis. Pour pouvoir continuer à les facturer, les médecins doivent attester d'un diplôme de formation continue (au choix). Pour cela, ils disposent des programmes de formation continue des 45 titres de spécialiste. Grâce à la "formation continue élargie», chaque programme offre la possibilité de suivre une formation continue dans les spécialisations de chacune des prestations de droits acquis sollicitées. Jusqu'à la nouvelle réglementation du concept de valeur intrinsèque, les assureurs acceptent que les médecins fassent valoir leur diplôme de formation continue à la place de la formation continue exigée pour chacune des positions de droits acquis. Ces dernières sont automatiquement prolongées conformément à la durée de validité du diplôme de formation continue.

\section{Recertification d'attestations de formation complémentaire?}

La formation continue pour les attestations de formation complémentaire suit d'autres règles, indépendantes des diplômes de formation continue des 45 titres de spécialiste: la plupart des attestations de formation complémentaire connaissent ce qu'on appelle une recertification dont les délais et les modalités sont décrits dans les programmes de formation respectifs. Le manquement aux prescriptions de recertification peut conduire à la perte de l'attestation. au cours d'une période de formation continue de trois ans. Chaque programme définit une formation continue essentielle spécifique de 25 crédits par an. Les sociétés de discipline médicale peuvent définir différentes catégories de formation et fixer le nombre maximal de crédits acceptés par catégorie. Les médecins peuvent faire valider 25 crédits au plus en tant que formation continue élargie. Ils peuvent ainsi suivre une formation continue hors de la discipline choisie. On entend par formation continue élargie toutes les sessions de formation pour lesquelles des crédits sont octroyés par une autre société de discipline médicale, une société cantonale de médecine ou la FMH. Chaque société de discipline médicale répond à toute question en lien avec le devoir de formation continue dans sa spécialité.

\section{Le diplôme de formation continue}

La plateforme de formation continue de l'ISFM (www. siwf.ch $\rightarrow$ Formation continue; cf. fig. 2) est la manière la plus simple d'obtenir le diplôme de formation continue. Cette plateforme vous permet d'enregistrer continuellement vos activités dans un protocole personnel et d'imprimer vous-même votre diplôme au terme de la période de formation continue de trois ans - à condition bien sûr d'avoir obtenu les 150 crédits exigés et de les avoir fait valider par la société de discipline médicale compétente. Ceci n'est toutefois possible que lorsque la discipline est mentionnée sur la plateforme de formation continue. Dans tous les autres cas, le médecin doit contacter la société de discipline médicale dont il souhaite acquérir le diplôme de formation continue. Ce dernier est exclusivement destiné aux membres de la FMH. Les non-membres obtiennent une attestation équivalente certifiant que le devoir de formation continue et la formation continue exigée pour les prestations de droits acquis ont été accomplis.

\section{Perspectives}

L'allègement des travaux administratifs profitera à tous les médecins qui pourront ainsi se concentrer davantage sur le cœur de leur métier, la prise en charge des patients. D'autres efforts seront nécessaires pour améliorer la qualité de la formation continue avec, comme objectif final, l'accroissement de la compétence professionnelle. C'est dans cette optique que se place la saisie centralisée de toutes les offres de formation continue en Suisse, liée à un octroi efficace de crédits et à une évaluation en ligne des sessions de formation continue.

\section{Questions fréquentes}

Vais-je perdre mon titre de spécialiste si je ne fais pas de formation continue?

Non, la formation continue est un devoir professionnel dont le manquement peut être sanctionné par les autorités sanitaires cantonales par un blâme ou une 
amende pouvant aller jusqu'à 20000 francs. Le retrait du titre de spécialiste n'est pas possible.

\section{Qui a l'obligation d'accomplir la formation continue?}

Conformément à l'art. 9 de la Réglementation pour la formation continue (RFC), tous les détenteurs d'un titre postgrade fédéral ou d'un titre postgrade étranger reconnu (aussi celui de «Médecin praticien») sont tenus d'accomplir leur formation continue, aussi longtemps qu'ils exercent une activité médicale en Suisse. Les médecins qui se trouvent en formation postgraduée en vue d'un titre de spécialiste ne sont pas soumis à cette exigence, ainsi que les médecins en formation qui possèdent déjà un titre de formation postgraduée.

Je me rends aux Etats-Unis pour travailler deux ans dans la recherche. Suis-je tenu d'accomplir une formation continue?

Non, vous n'êtes pas tenu d'accomplir une formation continue pendant cette période. Le devoir de formation continue s'applique uniquement aux médecins exerçant une activité médicale en Suisse (art. 9 RFC). Le devoir de formation continue ne reprendra vigueur qu'à votre retour en Suisse. Vous pouvez soit commencer une nouvelle période de formation continue de trois ans, soit faire valoir une réduction de deux ans de la formation continue obligatoire pour votre séjour à l'étranger. Il faut préciser que les séjours de courte durée à l'étranger (inférieurs à un an) ne suffisent pas pour suspendre le devoir de formation continue.

Je travaille dans le secteur administratif de Swissmedic en tant que médecin porteur d'un titre de spécialiste en médecine interne.

Suis-je tenu d'accomplir une formation continue? Le devoir de formation continue concerne tous les médecins qui exercent une «activité médicale». Une telle activité existe lorsque vous examinez, conseillez ou soignez des patients sous une forme quelconque. Une activité administrative ou de recherche sans contact avec des patients n'entre pas dans cette catégorie.

\section{Que se passe-t-il si je n'acquiers pas un diplôme} de formation continue?

L'acquisition du diplôme de formation continue n'est pas une obligation stipulée par la loi. L'important est que vous accomplissiez autant de formation continue que prescrit. Cela ne concerne en aucun cas votre titre de spécialiste. Ne pas posséder de diplôme de formation continue entraîne toutefois les inconvénients suivants:

- en cas de contrôle, vous devez convaincre les autorités cantonales concernées au moyen de documents appropriés que la formation continue que vous avez accomplie correspond au standard habituel. Il en va de même lors d'un procès éventuel de responsabilité civile.

- sans diplôme ou sans attestation de formation continue dans le registre doctorfmh.ch, les assureurs peuvent refuser la prise en charge des positions de droits acquis. Le cas échéant, vous seriez contraint de confirmer, dans la banque de données sur la valeur intrinsèque, la formation continue spéciale pour les prestations de droits acquis et l'attester au moyen de documents adéquats [2].

\section{Quel est le coût d'un diplôme de formation continue?}

La reconnaissance de sessions de formation continue et le contrôle de la formation continue sont effectués par la société de discipline médicale concernée, laquelle perçoit des taxes en fonction des dépenses occasionnées. Pour les membres d'une société de discipline médicale, cette taxe est généralement incluse dans la cotisation annuelle. Pour les non-membres, le diplôme valable pour trois ans ne devrait pas coûter plus de 150 francs. Des taxes plus élevées devront être approuvées par le Comité de l'ISFM.

\section{Comment puis-je accomplir une formation} continue dans le cadre de la médecine complémentaire si ma société de discipline médicale ne reconnaît aucune session de formation correspondante?

Dans la Réglementation sur la formation continue, la médecine complémentaire fait l'objet d'une disposition spéciale: les cinq sociétés qui gèrent une attestation de formation complémentaire (ASA, ASMOA, SSMH, SANTH, SMGP) peuvent reconnaître des sessions de formation et octroyer des crédits correspondants qu'il est possible de faire valider dans le cadre de la formation continue élargie.

Puis-je faire valoir la formation continue que j'accomplis dans le cadre d'une attestation de formation complémentaire également pour le diplôme de formation continue?

Seules les sessions de formation pour lesquelles des crédits sont octroyés par une société de discipline médicale (45 titres de spécialiste), une société cantonale de médecine ou la FMH peuvent être prises en compte pour le diplôme de formation continue. Les 5 sociétés de médecine complémentaire ASA, ASMOA, SSMH, SANTH, SMGP constituent une exception. Les sessions de formation d'autres organisations médicales doivent être reconnues au moins par une société de discipline médicale. Dès que c'est le cas, cette session peut être prise en compte dans le cadre de la formation continue élargie pour les 44 autres diplômes de formation continue.

Je suis porteur du titre de spécialiste en médecine générale et j'exerce principalement dans le domaine gynécologique. Je dispose des positions 


\section{La formation continue en 10 points}

1. Le devoir de formation continue est un devoir professionnel ancré dans l'art. 40 de la Loi sur les formations médicales (LPMéd). La surveillance et les contrôles incombent aux autorités cantonales concernées.

2. L'ISFM offre un diplôme de formation continue conjointement avec les 45 sociétés de discipline médicale représentant un titre de spécialiste. Ce diplôme permet à son détenteur:

- d'attester qu'il a accompli la formation continue stipulée par la LPMéd;

- d'attester auprès des assureurs qu'il a accompli la formation continue exigée pour les prestations de droits acquis.

3. Conformément à la Réglementation pour la formation continue (RFC), les médecins doivent acquérir au moins un diplôme de formation continue dans le domaine correspondant à leur activité professionnelle principale du moment.

4. Chaque programme de formation continue des 45 sociétés de discipline médicale fait la distinction entre les catégories suivantes:

- formation continue essentielle ( 25 crédits)

- formation continue élargie ( 25 crédits)

- étude personnelle (30 heures, non contrôlées)

La formation continue obligatoire comprend ainsi 80 heures de formation continue par an. Les médecins en activité doivent attester 150 crédits (dont au moins 75 crédits de formation continue essentielle) tous les trois ans.

5. Les médecins qui facturent des positions de droits acquis dans le cadre du TARMED doivent suivre une formation continue aussi dans ces domaines, conformément au concept de la valeur intrinsèque. La formation continue pour les positions de droits acquis peut être accomplie dans le cadre de la formation continue élargie de 75 crédits sur une période de 3 ans.

6. Le diplôme de formation continue doit être renouvelé tous les 3 ans.

7. Si vous n'acquérez pas (ne voulez pas acquérir) un diplôme de formation continue valable, vous avez l'obligation de déclarer la formation continue pour les prestations de droits acquis de manière individuelle sur myfmh, position par position (www.myfmh.ch) [2].

8. La recertification (formation continue) d'attestations de formation complémentaire a lieu d'après le programme de formation complémentaire concerné et est indépendante des 45 diplômes de formation continue des titres de spécialiste.

9. Vous trouverez sur notre site Internet www.siwf.ch, à la rubrique «Formation continue», les 45 programmes de formation continue, les interlocuteurs des sociétés de discipline médicale concernées et toutes les autres informations.

10. La plateforme de la formation continue (www.siwf.ch $\rightarrow$ Formation continue) vous permet d'enregistrer continuellement vos activités et d'imprimer vousmême le diplôme de formation continue après obtention des 150 crédits exigés (uniquement pour les spécialités qui y figurent).

de droits acquis correspondantes. Puis-je acquérir le diplôme de formation continue de la Société suisse de gynécologie et d'obstétrique et attester ainsi de la formation continue pour les prestations de droits acquis?

Comme vous exercez principalement dans le domaine de la gynécologie et de l'obstétrique, vous avez intérêt à choisir le programme de formation continue de la Société suisse de gynécologie et d'obstétrique. Mais en vertu de l'art. 12 de la RFC, le diplôme de formation continue n'est remis qu'aux porteurs du titre correspondant. Si vous validez 150 crédits, vous avez droit à une attestation de formation continue équivalente qui vous servira entre autres à attester de la for- mation continue pour les prestations de droits acquis exigée.

Je suis porteur de deux titres de spécialiste en médecine interne et en cardiologie. Dois-je obtenir le diplôme de formation continue dans ces deux domaines?

Non. Vous pouvez vous limiter au programme de formation continue qui correspond le plus à l'activité professionnelle que vous exercez. Vous êtes bien évidemment libre de suivre deux programmes de formation continue. Cette double formation n'entraîne pas une importante surcharge de travail puisque la formation continue essentielle d'un des programmes est automatiquement considérée comme la formation continue élargie de l'autre. Dès que vous avez accompli les formations continues essentielles spécifiques des deux disciplines sans chevauchement, vous avez droit aux deux diplômes de formation continue.

Je suis porteur d'un titre de spécialiste en médecine interne avec une formation approfondie en gériatrie. Existe-il un diplôme de formation continue pour cette spécialisation? Non. Depuis la révision de la Réglementation sur la formation continue du 26 mai 2010, seuls 45 programmes de formation continue sont proposés, soit un par titre de spécialiste. Cependant, la formation continue en gériatrie est prise en compte sans limitation de contenu pour le diplôme de formation continue en médecine interne générale.

Je suis mère de deux enfants et travaille à $50 \%$ dans un cabinet médical. Dois-je accomplir I'intégralité de la formation continue?

Oui. Un taux d'occupation partiel ne donne pas droit à une réduction de la formation continue obligatoire. La formation continue a pour objectif de garantir la qualité des prestations et de maintenir la compétence médicale, une garantie qu'il convient d'apporter également lors d'une activité à temps partiel.

\section{J'ai interrompu mon activité médicale pendant} un congé maternité de six mois. Dois-je valider moins de crédits pour le diplôme de formation continue?

Oui. Des interruptions de l'activité médicale dépassant six mois dans la période de formation de trois ans entraînent une réduction pro rata des crédits exigés. Ce même principe s'applique aux séjours à l'étranger ou à d'autres interruptions de l'activité médicale en Suisse.

\section{Références}

1 Hänggeli C, Kappeler O. Formation continue pour les positions de droits acquis. Bull Méd Suisses. 2006;87(18):768-71

2 Hänggeli C. Possédez-vous un diplôme de formation continue? Bull Méd Suisses. 2009;90(49): 1906-8. 